CASE REPORT

\author{
R. Manara \\ V. Citton \\ M. Rossetto \\ A. Padoan \\ D. D'Avella
}

\section{Hypophyseal Triplication: Case Report and Embryologic Considerations}

SUMMARY: Hypophyseal triplication is malformation that has not been described previously. We present a child with midline abnormalities who underwent epignathus excision at birth. Brain MR imaging revealed 2 paired lateral pituitary glands and an oval midline gland, each with an independent stalk, connected to a thickened third ventricle floor. Because malformations represent a failure in embryogenesis, this case may provide interesting clues on the normal development of the hypophysis.
D uplication of the pituitary gland is an extremely rare malformation; most patients do not survive beyond infancy because of the severity of associated abnormalities. ${ }^{1}$ Several cases present epipharyngeal teratoma, midline palatal cleft, or spinal malformations, thus revealing the failure of development of multiple midline structures. ${ }^{2-4}$ Besides the classic form with 2 complete pituitary glands located in the lateral recesses of the pituitary fossa, the spectrum of hypophyseal malformation includes incomplete duplication affecting the anterior pituitary gland or the pituitary stalk. ${ }^{5}$ The floor of the third ventricle usually appears thickened, and this feature has been differently interpreted as a hamartoma or as the result of either the duplication of the hypothalamic nuclei, or the fusion of the mammillary bodies and tuber cinereum into a single midline mass. ${ }^{1}$

We report the MR imaging findings of an hypophyseal triplication in a 7-year-old girl with concomitant craniofacial and spinal abnormalities. Because the embryogenesis of the pituitary gland is still controversial, ${ }^{1,3,4}$ morphologic and signal intensity characteristics of the 3 pituitary glands have been considered to gain insight into the normal development of the hypophyseal-hypothalamic axis.

\section{Case Report}

A 7-year-old first child of nonconsanguineous parents, with a normal female karyotype and a known occult spinal dysraphism, was referred to our center for worsening of scoliosis and the onset of micturition.

According to her clinical history, she was born at 36 weeks' gestation by cesarean delivery because of a massive oropharyngeal epignathus detected by sonography examination at the fifth month of gestation. At birth, to safely manage airway obstruction, an ex utero intrapartum treatment (EXIT) procedure was performed, which consisted of maintaining placental support until the airway was secured. Fifteen days later, the protruding epignathus teratoma was removed, while a concomitant midline palate and lip cleft was repaired by serial maxillofacial surgery.

In our center, the patient underwent an MR imaging examination of the lumbosacral spine and the brain. The spinal MR imaging confirmed sacral spina bifida with butterfly vertebra at $\mathrm{S} 1$ and hemispondyls in the inferior part of the sacrum. Moreover, an intradural li-

Received December 10, 2008; accepted after revision December 23.

From the Neuroradiologic Unit (R.M., V.C.), University Hospital of Padua, Padua, Italy; and Department of Neurosurgery (M.R., A.P., D.D.), University of Padua, Padua, Italy.

Please address correspondence to Renzo Manara, MD, Neuroradiologic Unit, University Hospital of Padua, Via Giustiniani 5, 35128 Padua, Italy; e-mail: renzo.manara@ sanita.padova.it

DOI 10.3174/ajnr.A1520 poma tethering the spinal cord to its anterior-superior surface was detected.

Brain MR imaging confirmed the midline palatal cleft and disclosed concomitant multiple pituitary glands (Fig 1C). Two clearly showed adenohypophyseal and neurohypophyseal components and were situated almost specularly in the lateral recesses of the pituitary sella (Figs $1 A, 2 A, 2 C$ ). A midline third gland appeared as a partially intrasellar ovoid mass, characterized by a homogeneous signal intensity, intermediate between gray and white matter (Fig 1B). Each gland had an independent stalk arising from the floor of the third ventricle, which appeared diffusely thickened (Fig $2 B$ ), as previously described in pituitary duplication. ${ }^{1,3}$ The olfactory bulbs, the anterior commissure, the ventricles, and the corpus callosum were normal; the intracranial segment of the vertebral arteries was tortuous, but no signs of basilar fenestration or duplication were observed. A thin lipoma surrounded the corpus callosum, and a slight hypertelorism was noted.

\section{Discussion}

Duplication of the hypophyseal gland has been rarely described, often in association with several midline malformations. ${ }^{3,5}$ Most children do not survive beyond the neonatal period because of the severity of associated malformations. ${ }^{1}$ Our patient presented with, among other midline cranial and extracranial abnormalities, an epignathus teratoma, diagnosed by fetal sonography investigation, which required a complex procedure (EXIT) to provide the patency of airway for subsequent surgical removal. ${ }^{6}$ Nevertheless, the outstanding interest in this case is not because of the numerous concomitant abnormalities involving both head and spine, which simply represent the expression of a multisite failure of midline structures development, nor in the number of pituitary glands ultimately recognized in this patient, which further broadens the spectrum of anterior midline defects. ${ }^{3,7}$ This case attracted our attention because the presence of 3 pituitary glands and their signal intensity characteristics seem to provide interesting hints on the embryologic normal development of the hypothalamic-pituitary axis.

Because a histologic confirmation or postcontrast images are not available, the midline mass could be also interpreted as a pedunculated hypothalamic hamartoma, a rare non-neoplastic congenital heterotopia of gray matter, which can be found in midline cleft defects. The absence of gelastic seizures and of precocious puberty and the signal intensity characteristic of the mass (relatively hypointense on proton densityweighted images and hyperintense on T1-weighted images compared with gray matter) do not support this hypothesis.

Currently, there are 2 fairly different theories on the origin 


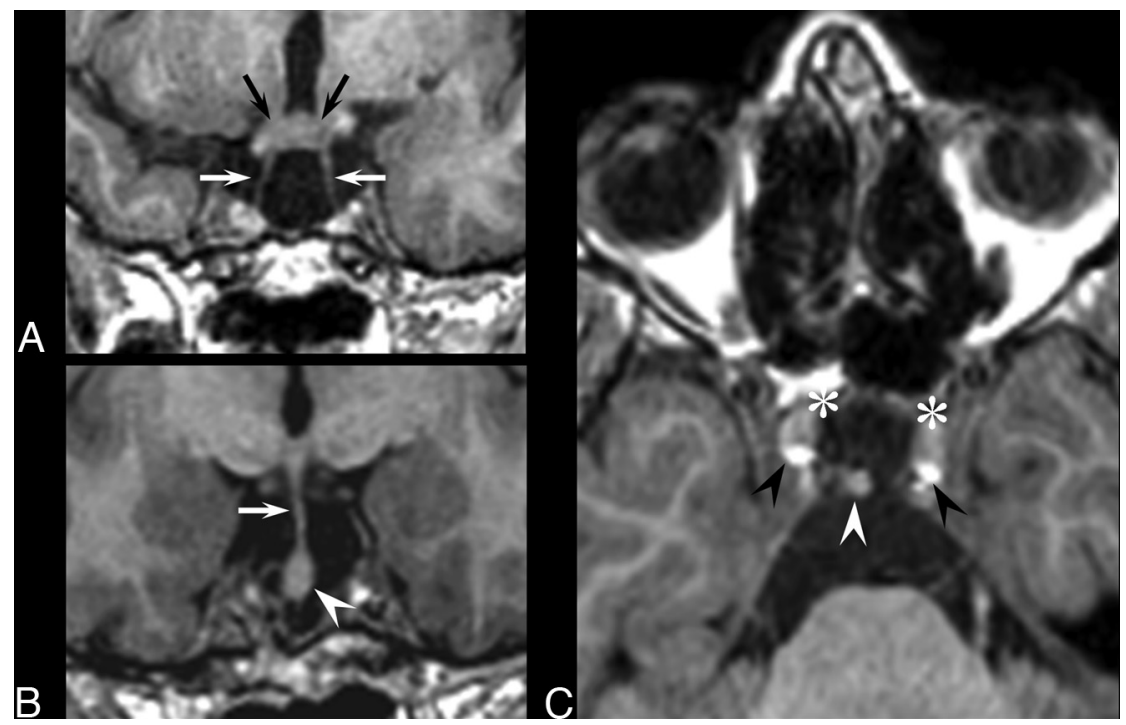

Fig 1. Unenhanced T1-weighted multiplanar reformatted MR images. A, Coronal image showing paired infundibula (white arrows) extending inferiorly to 2 pituitary glands symmetrically located in the lateral recesses of the pituitary fossa; the hypothalamus appears grossly thickened (black arrows). $B$ Coronal image along the pituitary stalk (white arrow) of the median aspect of the hypophysis, which appears as an oval, hyperintense-to-gray matter, homogeneous mass partially housed in the pituitary fossa (white arrowhead). Similar features might be found also in pedunculated hamartomas, which are usually characterized by the presence of clinical and laboratory signs of precocious puberty and by $\mathrm{T} 1$ isointensity compared with gray matter. $C_{r}$ In this oblique axial image, the 3 pituitary glands are contemporaneously visible; the paramedian glands present both the adenohypophysea component (white stars) and the neurohypophyseal bright spot (black arrowheads), whereas the midline hypophysis (white arrowhead) is homogeneously isointense.

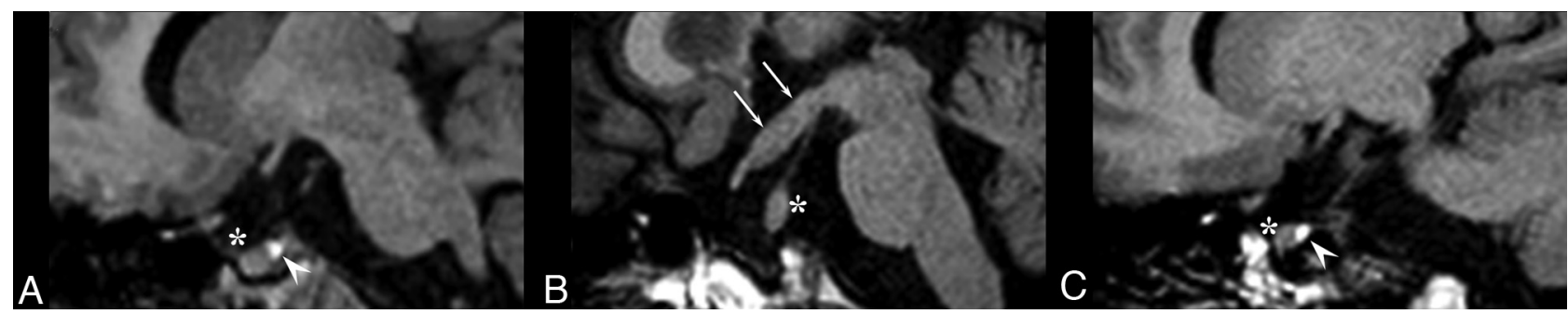

Fig 2. $A, C$, Paramedian sagittal T1-weighted images show 2 different pituitary glands, each with a posterior bright spot (white arrowhead) and an anterior pituitary lobe (white star). $B$ Midline sagittal T1-weighted image revealing a thickened third ventricle floor (white arrows) and an homogeneous, isointense median mass (white star) with its independent stalk.

of the pituitary gland. According to the classic theory, ${ }^{1,3,5}$ the hypophysis is believed to arise from 2 distinct structures, namely the diencephalic neuroectoderm (posterior lobe) and the oral ectoderm (anterior lobe). At approximately 42 days of gestation, a diverticulum originating from the stomodeum fuses with the downward extending diencephalic bud, both finally housed in the pituitary fossa. In contrast to the traditional view, Gilbert (1934) found that in mammalian species, both the anterior and posterior part of the gland originate from the ventral neural ridge. ${ }^{1}$ In this model, resumed by Morton in $1957^{4}$ in a case report of pituitary duplication, the gland is supposed to arise entirely from neuroectodermal tissue without the primary involvement of the Rathke pouch. These 2 theories primarily differ in the role of the Rathke pouch, which seems necessary for development of the adenohypophysis in the former, whereas it is not required in the latter. When a midline cleft is present, the Rathke pouch can reach the above developing gland only aside the cleft itself. In our case, both the lateral glands presented signal intensity characteristics consistent with the coexistence of neurohypophyseal and adenohypophyseal components (Figs $1 A, 1 C$, $2 A, 2 C)$. It is interesting to note that the third gland, situated above the midline cleft, presented a homogeneous MR signal intensity, isointense to the hypothalamic floor, consistent with the presence of the neurohypophyseal component only (Figs $1 B, 2 B)$. According to Gilbert's theory, there should be no reason for the absence of both neurohypophyseal and adenohypophyseal components in the midline gland because the
Rathke pouch does not contribute to the development of any of them. Hence, the coincidence of 2 "normal" paired lateral pituitary glands with a third "incomplete" hypophysis, just above a midline cleft hampering the normal development of the Rathke pouch, strongly suggests its pivotal role in the normal development of the hypothalamic-pituitary axis.

In conclusion, we present a patient affected by multiple midline defects with triplication of the pituitary gland. This extraordinary condition seems to support the hypothesis of a Rathke pouch contribution to the genesis of the pituitary gland, thus contrasting the alternative hypothesis of an entirely neuroectodermal origin of the hypothalamic-pituitary axis.

\section{References}

1. Tortori-Donati P. Pediatric Neuroradiology, Brain. New York: Springer-Verlag; 2005:855-91

2. Burke M, Zinkovsky S, Abrantes MA, et al. Duplication of the hypophysis. Pediatr Neurosurg 2000;33:95-99

3. Kollias SS, Ball WS, Prenger EC. Review of the embryologic development of the pituitary gland and report of a case of hypophyseal duplication detected by MRI. Neuroradiology 1995;37:3-12

4. Morton WR. Duplication of the pituitary and stomatodaeal structures in a 38-week male infant. Arch Dis Child 1957;32:135-41

5. Kandpal H, Seith A, Philip J, et al. Partial duplication of the hypophysis in adult patients: report of 2 cases. J Comput Assist Tomogr 2007;31:365-67

6. Midrio P, Grismondi G, Meneghini L, et al. The EX-utero Intrapartum Technique (EXIT) procedure in Italy. Minerva Ginecol 2001;53:209-14

7. Loddenkemper T, Friedman NR, Ruggieri PM, et al. Pituitary stalk duplication in association with Moya moya disease and bilateral morning glory disc anomaly_broadening the clinical spectrum of midline defects. J Neurol 2008 ; 255:885-90 